\title{
Respect par les Canadiens de 10 à 17 ans des Directives canadiennes en matière de mouvement sur 24 heures pour les enfants et les jeunes
}

Ian Janssen, Ph. D. (1,2); Karen C. Roberts, M. Sc. (3); Wendy Thompson, M. Sc. (3)

Cet article a fait l'objet d'une évaluation par les pairs.

Diffuser cet article sur Twitter

\section{Résumé}

Introduction : Le document Directives canadiennes en matière de mouvement sur 24 heures pour les enfants et les jeunes publié en 2016 contient des recommandations quant à l'activité physique quotidienne d'intensité moyenne à élevée, au temps de loisir passé devant un écran et au sommeil chez les jeunes de 5 à 17 ans. Cette étude a pour objectif d'estimer le pourcentage de Canadiens de 10 à 17 ans qui respectent ces recommandations.

Méthodologie : Nous avons analysé un échantillon national représentatif de 22115 jeunes. Les données sur le comportement actif ont été autodéclarées. Le respect des recommandations des Directives repose sur les éléments suivants : accumulation d'au moins 60 minutes par jour d'activité physique d'intensité moyenne à élevée, pas plus de 2 heures par jour de temps de loisir passé devant un écran et de 9 à 11 heures de sommeil continu par nuit pour les jeunes de 10 à 13 ans ou de 8 à 10 heures de sommeil continu par nuit pour les jeunes de 14 à 17 ans.

Résultats : Seulement $3 \%$ des jeunes de l'échantillon respectaient les trois recommandations clés. Vingt-cinq pour cent respectaient deux recommandations, $51 \%$ en respectaient une et $21 \%$ n'en respectaient aucune. La recommandation en matière de sommeil était la plus respectée par les enfants et les jeunes (66\%), suivie de celle en matière d'activité physique d'intensité moyenne à élevée (35\%) et de celle relative au temps passé devant un écran $(8 \%)$.

Conclusion : Une faible proportion (moins de $3 \%$ ) de Canadiens de 10 à 17 ans respectent les trois principales recommandations des Directives canadiennes en matière de mouvement sur 24 heures pour les enfants et les jeunes.

Mots-clés : activité physique, temps passé devant l'écran, sommeil, enfants, jeunes

\section{Introduction}

Un manque d'activité physique ${ }^{1,2}$, un comportement sédentaire excessif (en particulier le temps de loisir passé devant un écran) $)^{3,4}$ et un manque de sommeil ${ }^{5,6}$ sont associés à plusieurs indicateurs de santé physique, mentale et sociale chez les enfants d'âge scolaire et les jeunes. Le Canada a établi des lignes directrices de santé publique distinctes pour l'activité physique $^{7}$ et pour le comportement sédentaire ${ }^{8}$.
La U.S. National Sleep Foundation a établi des lignes directrices sur la durée du sommeil ${ }^{9}$, et ces dernières sont approuvées par les spécialistes canadiens du sommeil chez les enfants ${ }^{5}$. L'existence de ces trois ensembles de directives montre que l'activité physique, le comportement sédentaire et le sommeil ont été jusqu'à présent considérés comme des facteurs indépendants. Les chercheurs commencent à intégrer que ces trois facteurs sont liés et qu'ils doivent être conjointement analysés ${ }^{10-13}$. Étant donné que

\section{Points saillants}

- Cette étude a pour objectif d'estimer le pourcentage de Canadiens de 10 à 17 ans qui respectent les recommandations des Directives canadiennes en matière de mouvement sur 24 heures pour les enfants et les jeunes.

- Seulement $3 \%$ des enfants et des jeunes de l'échantillon respectaient les recommandations en matière d'activité physique d'intensité moyenne à élevée, de temps passé devant un écran et de sommeil.

- La recommandation en matière de sommeil était la plus respectée par les enfants et les jeunes (par $66 \%$ ), suivie de celle en matière d'activité physique d'intensité moyenne à élevée (35\%) et de celle relative au temps passé devant un écran (8 \%).

ces trois facteurs sont mutuellement exclusifs, et que le temps consacré à ces trois facteurs dans une journée doit totaliser 24 heures, l'activité physique, le comportement sédentaire et le sommeil sont codépendants. Autrement dit, le temps consacré à un de ces facteurs affecte forcément le temps consacré à au moins un des deux autres facteurs.

C'est en raison de cette codépendance de l'activité physique, du comportement sédentaire et du sommeil qu'un important groupe de chercheurs et d'utilisateurs de connaissances ont récemment publié les Directives canadiennes en matière de mouvement sur 24 heures pour les enfants et les

Rattachement des auteurs :

1. École de kinésiologie et d'études sur la santé, Université Queen's, Kingston (Ontario), Canada

2. Département des sciences de la santé publique, Université Queen's, Kingston (Ontario), Canada

3. Agence de la santé publique du Canada, Ottawa (Ontario), Canada

Correspondance : Ian Janssen, École de kinésiologie et d'études sur la santé, Université Queen’s, Kingston (Ontario) K7L 3N6; tél. : 613-533-6000, poste 78631; courriel : ian.janssen@queensu.ca 
jeunes : une approche intégrée regroupant l'activité physique, le comportement sédentaire et le sommeil ${ }^{14}$. Nous y ferons référence ici par l'expression abrégée "Directives en matière de mouvement sur 24 heures » ou " Directives ». Ces Directives ont été préparées sous la direction de la Société canadienne de physiologie de l'exercice, avec le soutien et l'approbation du Centre hospitalier pour enfants de l'est de l'Ontario, du Conference Board du Canada, de l'Agence de la santé publique du Canada et de ParticipACTION. Elles contiennent des recommandations précises sur le temps que devraient consacrer les jeunes de 5 à 17 ans à l'activité physique d'intensité moyenne à élevée (APIME), soit $60 \mathrm{~min}$ utes par jour ou plus, au temps de loisir devant un écran, soit 2 heures par jour ou moins, et au sommeil, soit de 9 à 11 heures par nuit pour les enfants de 5 à 13 ans et de 8 à 10 heures par nuit pour les jeunes de 14 à 17 ans, pour assurer une croissance saine. Le respect de ces recommandations est associé à plusieurs résultats cliniques comme la composition corporelle, la forme physique, le rendement scolaire et l'apprentissage, la régulation émotionnelle, les comportements prosociaux, la capacité cardiovasculaire et métabolique ainsi que la qualité de vie générale. En établissant ces recommandations, les Directives en matière de mouvement sur 24 heures fixent des cibles de surveillance mesurables et offrent un cadre d'action aux professionnels de la santé, aux chercheurs, aux décideurs et au grand public. De plus, leur existence signifie que mettre l'accent sur un seul des trois facteurs constitue une stratégie désuète, car une personne qui respecte une recommandation n'a pas nécessairement un profil comportemental sain en ce qui concerne les autres. Par exemple, un enfant qui fait suffisamment d'APIME mais qui passe trop de temps devant un écran et qui ne dort pas suffisamment n'a pas un profil comportemental idéal en matière de mouvement.

Maintenant que les Directives en matière de mouvement sur 24 heures sont publiées, il est important de récolter des données sur la proportion d'enfants et de jeunes Canadiens qui respectent les trois recommandations relativement au mouvement. Cette information peut en effet servir à établir des programmes et des politiques sur l'adoption de comportements sains en matière de mouvement. C'est dans la récolte de ces données que réside le principal objectif de notre étude. Les objectifs secondaires sont d'estimer les proportions d'enfants et de jeunes qui respectent chacune des recommandations indépendamment ainsi que les proportions pour les différentes combinaisons de recommandations (p. ex. activité physique et sommeil, mais pas temps passé devant un écran). Nous avons eu l'occasion de réaliser ces objectifs grâce à l'Enquête sur les comportements liés à la santé des enfants d'âge scolaire (ECSEAS), qui porte sur un vaste échantillon représentatif de jeunes Canadiens de $6^{\mathrm{e}}$ année au secondaire 4 .

\section{Méthodologie}

\section{Conception et échantillon de l'étude}

Notre étude repose sur les données canadiennes du cycle 2013-2014 de l'ECSEAS, une enquête collaborative internationale de l'Organisation mondiale de la santé (OMS) portant sur les étudiants de la $6^{\mathrm{e}}$ année au secondaire $4^{15,16}$. Le cycle 2013-2014 réalisé au Canada a suivi le protocole d'échantillonnage international. Ainsi, des classes de 349 écoles ont été sélectionnées au moyen d'une technique de probabilité pondérée, afin d'assurer une représentation proportionnelle en fonction du lieu, de la langue, de la religion et de la taille de la collectivité. Les jeunes inscrits à un programme pour enfants ayant des besoins particuliers, ceux vivant dans une réserve ou ne fréquentant pas une école publique ont été exclus, soit au total moins de $7 \%$ des jeunes Canadiens ${ }^{16}$. Ce sont ainsi $77 \%$ des étudiants initialement sélectionnés qui ont participé à l'étude, ce qui a consisté à remplir un questionnaire de 45 minutes. Le consentement a été obtenu auprès des étudiants, des parents ou tuteurs, des écoles et des commissions scolaires. L'étude a été approuvée sur le plan éthique par le General Research Ethics Board de l'Université Queen's (dossier n 6010236).

Les questions incluses dans le questionnaire de l'ECSEAS sont continuellement élaborées, validées et soumises à des tests pilotes par le réseau international de l'ECSEAS, et au Canada par les chercheurs canadiens de l'ECSEAS ${ }^{15,16}$. Même si, dans la plupart des cas, les résultats de ces études de validation et tests pilotes ne sont pas publiés, le protocole international de l'ECSEAS exige des données probantes démontrant que les questions ont des propriétés psychométriques acceptables et qu'elles sont comprises par les élèves de la $6^{\mathrm{e}}$ à la $10^{\mathrm{e}}$ année.
Une faible proportion ( $\mathrm{n}=606 ; 2,0 \%$ ) de l'échantillon initial de 30153 étudiants a rempli un questionnaire abrégé qui n'incluait pas les questions sur le sommeil. De plus, 369 étudiants $(1,2 \%)$ ne correspondaient pas à la tranche d'âge (p. ex. élève de secondaire 5 dans une classe de secondaire 4). L'échantillon admissible pour notre étude était donc de 29178 enfants et jeunes de 10 à 17 ans. Parmi eux, 67 $(0,2 \%)$ ont été exclus car il manquait des données sur leur âge ou leur sexe, puis 4429 étudiants (15,1\%) ont été exclus car il manquait des données pour au moins l'une des questions sur le sommeil ou parce que la durée de leur sommeil durant la semaine ou la fin de semaine était supérieure à trois écarts-types (nous avons alors présumé que ces données anormales étaient attribuables à des erreurs de consignation). Enfin, 1772 étudiants (6,1\%) ont été exclus parce qu'ils n'avaient pas répondu à au moins l'une des questions sur l'activité physique, et 975 étudiants (3,3 \%) ont été exclus car ils n’avaient pas répondu à au moins l'une des questions sur le temps passé devant un écran. L'échantillon final pour notre étude était de 22115 enfants et jeunes. Nous présentons dans le tableau 1 une comparaison entre l'échantillon définitif et les 7063 participants exclus des analyses statistiques. Les différences relatives entre les groupes inclus et les groupes exclus étaient inférieures à $10 \%$ pour toutes les variables individuelles (comme l'âge, le sexe, l'origine ethnique), géographiques (province ou territoire, taille de la municipalité) et en lien avec le mouvement (sommeil, APIME, temps passé devant un écran).

\section{Directives en matière de mouvement sur 24 heures}

Le document décrivant les Directives et leur préparation explique comment elles doivent être interprétées dans une optique de surveillance ${ }^{14}$. On y indique que les conditions minimales suivantes doivent être satisfaites pour la surveillance : 1) de 9 à 11 heures de sommeil continu par nuit pour les enfants de 5 à 13 ans ou de 8 à 10 heures de sommeil continu par nuit pour les jeunes de 14 à 17 ans, 2) une accumulation d'au moins 60 minutes par jour d'APIME avec diverses activités aérobiques et 3) pas plus de 2 heures par jour de temps de loisir passé devant un écran. Il est précisé que ces trois conditions doivent être remplies en respectant la moyenne des heures quotidiennes calculée sur une semaine. 
TABLEAU 1

Caractéristiques descriptives des participants de l'ECSEAS 2013-2014 inclus dans les analyses statistiques et exclus

\begin{tabular}{|c|c|c|}
\hline Caractéristique & $\begin{array}{l}\text { Participants inclus } \\
\qquad \begin{array}{l}(\mathrm{n}=22115) \\
\text { n (IC à } 95 \%)\end{array}\end{array}$ & $\begin{array}{l}\text { Participants exclus } \\
\text { (n = } 7063) \\
\text { n (IC à } 95 \%)\end{array}$ \\
\hline Âge moyen & $14,1 \quad(13,9$ à 14,3$)$ & $14,1 \quad(13,9$ à 14,3$)$ \\
\hline Sexe (\% de filles) & 52,8 (51,2 à 54,5) & $45,2(42,7$ à 47,4$)$ \\
\hline Origine ethnique (\% de Caucasiens) & 76,0 (71,6 à 80,5) & $70,7(65,3$ à 76,0$)$ \\
\hline Immigration (\% nés au Canada) & $81,3 \quad(79,2$ à 83,4) & 77,5 (74,5 à 80,4) \\
\hline $\begin{array}{l}\text { Perception du degré d'aisance de la famille (\% } \\
\text { percevant un faible degré d'aisance) }\end{array}$ & $8,7 \quad(8,0$ à 9,3$)$ & $9,0 \quad(8,0$ à 10,1$)$ \\
\hline \multicolumn{3}{|l|}{ Région du Canada } \\
\hline Ouest (C.-B., Alb.) (\%) & $24,7 \quad(16,1$ à 33,3$)$ & $23,5(13,9$ à 33,2$)$ \\
\hline Prairies (Sask., Man.) (\%) & $7,8 \quad(4,3$ à 11,3$)$ & $7,2 \quad(3,7$ à 10,7$)$ \\
\hline Ontario (\%) & 42,8 (33,2 à 52,5) & $38,6(28,3$ à 48,9$)$ \\
\hline Québec (\%) & $19,1 \quad(8,7$ à 29,5$)$ & $21,3 \quad(10,0$ à 32,6$)$ \\
\hline Maritimes (N.-É., N.-B., Î.-P.-É.) (\%) & $3,4 \quad(1,7$ à 5,1$)$ & $7,4 \quad(1,2$ à 13,6$)$ \\
\hline Territoires (T.N.-O., Yn, Nun.) (\%) & $0,5 \quad(0,3$ à 0,7$)$ & $0,6 \quad(0,3$ à 0,8$)$ \\
\hline \multicolumn{3}{|l|}{ Type de municipalité } \\
\hline Rurale (< 1000 hab.) (\%) & $4,1 \quad(1,2$ à 7,1$)$ & $3,3 \quad(1,2$ à 5,5$)$ \\
\hline Petite ville (1 000 à 29999 hab.) (\%) & 36,7 (26,5 à 46,8) & $39,9(28,7$ à 51,0$)$ \\
\hline Ville moyenne (30 000 à 99999 hab.) (\%) & $18,7 \quad(11,2$ à 26,3$)$ & $19,0(10,1$ à 28,0$)$ \\
\hline Grande ville (100 000 à 499999 hab.) (\%) & 25,2 (16,9 à 33,5) & $21,8(13,3$ à 30,2$)$ \\
\hline Centre métropolitain ( $\geq 500000$ hab.) (\%) & $15,3 \quad(8,6$ à 22,0$)$ & $15,9 \quad(7,5$ à 24,4$)$ \\
\hline \multicolumn{3}{|l|}{ Comportements en matière de mouvement } \\
\hline Durée du sommeil (heures/nuit) & $9,0 \quad(8,9$ à 9,0$)$ & $(8,9$ à 9,1$)$ \\
\hline APIME (heures/semaine) & $5,6 \quad(5,4$ à 5,8$)$ & $(5,6$ à 6,1$)$ \\
\hline Temps d'écran (heures/jour) & $7,5 \quad(7,3$ à 7,7$)$ & $8,1 \quad(7,8$ à 8,5$)$ \\
\hline
\end{tabular}

Abréviations : APIME, activité physique d'intensité moyenne à élevée; ECSEAS, Enquête sur les comportements liés à la santé des enfants d'âge scolaire; hab., habitants; IC, intervalle de confiance.

Remarque : Les données présentées sont des moyennes (IC à $95 \%$ ) pour les variables continues et des prévalences (IC à $95 \%$ ) pour les variables nominales.

\section{Durée du sommeil}

On a demandé aux participants de consigner la durée habituelle d'une nuit de sommeil au cours de la semaine précédente, entre le moment où ils éteignaient la lumière le soir jusqu'au réveil le lendemain, et en distinguant les jours de semaine de ceux de fin de semaine. À partir de cette information, nous avons calculé la moyenne d'heures de sommeil de chaque participant et nous avons déterminé si les participants respectaient ou non la durée de sommeil recommandée (soit de 9 à 11 heures par nuit pour les enfants de 5 à 13 ans et de 8 à 10 heures par nuit pour les jeunes de 14 à 17 ans) ${ }^{14}$. Toute durée du sommeil qui ne respectait pas ces plages (même pour une minute) a été considérée comme n'étant pas conforme à ces recommandations. Les résultats de l'analyse de validité révèlent que les estimations de la moyenne d'heures de sommeil chez les jeunes calculée selon un processus d'autodéclaration sont comparables à la moyenne objectivement mesurée au moyen d'un actigraphe (respectivement 471 contre 461 minutes par nuit) ${ }^{17}$.

\section{Activité physique d'intensité moyenne à élevée (APIME)}

Les enfants et les jeunes accumulent leur APIME en pratiquant des activités physiques à l'école (p. ex. classe d'éducation physique), des sports organisés durant leurs temps libres et des activités d'extérieur pendant leurs temps libres ou en utilisant des moyens de transport actif (comme se rendre l'école en marchant ou à bicyclette) ${ }^{18}$. La durée totale d'APIME en classe et pendant les temps libres hors de l'école (notamment les programmes de sport et les activités extérieures) a été évaluée grâce aux réponses aux deux questions suivantes : "Pendant environ combien d'heures par semaine pratiques-tu, à l'école, des activités physiques qui t'essoufflent ou qui te donnent plus chaud que la normale? » et " À l'extérieur de l'école : Pendant environ combien d'heures par semaine pratiques-tu, pendant tes temps libres, des activités physiques qui t'essoufflent ou te font suer? ». Les choix de réponse étaient les suivants : " Aucune", "Environ 30 minutes ", "Environ 1 heure ", " Environ 3 heures ", "Environ 4 heures ", «Environ 5 heures », "Environ 6 heures » et «Environ 7 heures ». Le nombre d'heures de transport actif a été évalué avec les questions suivantes : "Habituellement, ton trajet pour te rendre à l'école ou pour en revenir se fait... " et "Combien de temps dure ton trajet entre l'école et la maison? ». Les participants qui n'ont pas répondu « en marchant » ou «à bicyclette » pour le mode de transport jusqu'à l'école ou pour en revenir n'ont pas été considérés comme accumulant du temps de transport actif. Pour les participants qui ont répondu « en marchant " ou « à bicyclette », leur nombre d'heures de transport actif par semaine a été calculé en multipliant la durée du trajet entre l'école et la maison par deux (pour l'aller et le retour) puis en multipliant la réponse par cinq (pour les cinq jours de la semaine). Nous avons ensuite additionné le nombre d'heures d'APIME en classe et à l'extérieur de l'école ainsi que le temps de transport actif puis nous l'avons divisé par sept pour obtenir la moyenne quotidienne d'APIME. Nous avons alors classé les participants en deux catégories en fonction de cette moyenne : ceux qui respectaient la recommandation en matière d'APIME (au moins 60 minutes par jour en moyenne) et ceux qui ne la respectaient pas (moins de 60 minutes par jour en moyenne).

Les analyses de fiabilité test-retest indiquent une correspondance de 0,80 ou plus entre les réponses répétées à la question sur le transport actif dans l'ECSEAS ${ }^{19}$. La correspondance entre les réponses répétées aux questions sur l'activité physique à l'école et pendant les temps libres variait entre $67 \%$ et $85 \%$ selon le sexe et le groupe d'âge $^{20}$. La fiabilité test-retest pour la 
participation à des programmes de sport était également élevée (kappa $=0,84)^{21}$.

Il est important de souligner que la recommandation relative à la moyenne quotidienne d'APIME est différente de la façon dont les précédentes études de surveillance canadiennes analysaient ce comportement, bien que cette recommandation en soi n'ait pas changé depuis la publication des anciennes directives relatives à l'activité physique $^{7}$. La plupart des études ont évalué le respect de la recommandation relative à l'APIME en fonction de l'atteinte d'une moyenne de 60 minutes par jour pendant 6 ou 7 jours de la semaine $e^{16,22,23}$, conformément aux recommandations du Canada et de l'OMS au moment où les directives sur l'activité physique ont été publiées ${ }^{7,24}$.

\section{Temps passé devant un écran}

Le temps passé à regarder des divertissements sur écran, à jouer à des jeux vidéo sédentaires et à utiliser des appareils électroniques à diverses autres fins a été évalué au moyen des questions suivantes : " Combien d'heures par jour, pendant tes temps libres, passes-tu à regarder la télévision, des vidéos (par exemple sur YouTube ou une plateforme semblable), des DVD et d'autres divertissements sur un écran? ", «Combien d'heures par jour, pendant tes temps libres, passes-tu à jouer à des jeux sur l'ordinateur, une console, une tablette (comme un iPad), un téléphone intelligent ou tout autre appareil électronique (à l'exclusion des jeux où tu dois bouger ou d'entraînement)? » et " Combien d'heures par jour, pendant tes temps libres, passestu à utiliser des appareils électroniques comme un ordinateur, une tablette (comme un iPad) ou un téléphone intelligent pour autre chose (p. ex. devoirs, courriels, Twitter, Facebook, clavardage, navigation sur Internet) ». Pour chaque question, les choix de réponse étaient les suivants : " Aucune ", " Environ 30 minutes ", " Environ 1 heure », " Environ 2 heures ", " Environ 3 heures », "Environ 4 heures », " Environ 5 heures », "Environ 6 heures », «Environ 7 heures ou plus ». Les questions ont été posées pour les jours de semaine et ceux de fin de semaine. La correspondance entre les réponses répétées aux questions sur le temps passé devant un écran dans l'ECSEAS était de moyenne à élevée, avec des coefficients Kappa variant entre 0,68 et $0,82^{25}$. Nous avons calculé le nombre moyen d'heures par jour et nous avons classé les participants en deux catégories : ceux qui respectaient la recommandation $(2,0$ heures par jour ou moins) et ceux qui ne la respectaient pas (plus de 2,0 heures par jour).

\section{Groupes d'âgé et sexe}

Nous comparé les sexes (10 480 garçons et 11664 filles) et les groupes d'âge. Pour la comparaison entre groupes d'âge, nous avons divisé les participants en deux catégories : ceux de 10 à 13 ans $(n=10243)$ et ceux de 14 à 17 ans (n = 11 901). Le point de partage entre ces deux groupes correspond au changement de durée de sommeil recommandée entre 13 et 14 ans $^{14}$.

\section{Analyses statistiques}

Nous avons effectué les analyses statistiques avec la version 9.4 de SAS (Cary, Caroline du Sud, États-Unis). Nous avons utilisé les procédures de sondage avec options de pondération et de regroupement pour tenir compte de la pondération de l'échantillon et de la nature regroupée (par classe) du questionnaire. Nous avons calculé les prévalences des participants respectant les recommandations des Directives en matière de mouvement sur 24 heures, différentes combinaisons de recommandations et divers nombres de recommandations. Nous avons mesuré les différences entre sexes et groupes d'âge en utilisant le test du chi carré Rao-Scott, qui nous a permis de contrôler le regroupement par niveau scolaire. Nous avons utilisé une valeur prédictive inférieure à 0,05 pour établir la signification statistique.

\section{Résultats}

Nous décrivons l'échantillon utilisé pour les analyses statistiques dans le tableau 1 et nous présentons des renseignements supplémentaires sur le pourcentage de participants respectant les recommandations en matière de sommeil, d'APIME et de temps passé devant un écran dans le tableau 2. Au sein de l'échantillon représentatif d'enfants et de jeunes de 10 à 17 ans, $66 \%$ respectaient la recommandation pour le sommeil, $35 \%$ celle pour l'APIME et $8 \%$ celle pour le temps passé devant un écran (tableau 2). Les proportions de garçons et de filles respectant la recommandation pour le sommeil étaient les mêmes. En revanche, davantage de garçons que de filles respectaient la recommandation d'APIME par jour et, à l'inverse, davantage de filles que de garçons respectaient la recommandation relative au temps passé devant un écran ( 2 heures ou moins par jour). Le pourcentage de jeunes de 10 à 13 ans et celui de jeunes de 14 à 17 ans respectant les recommandations pour le sommeil ou l'APIME étaient semblables, mais une plus grande proportion de jeunes de 10 à 13 ans respectaient la recommandation relative au temps passé devant un écran.

Comme l'illustre le tableau 3, $21 \%$ de l'échantillon ne respectaient aucune des trois recommandations. Ce pourcentage était plus élevé chez les filles que chez les garçons mais ne différait pas en fonction de l'âge. Environ $51 \%$ des participants respectaient l'une des recommandations, $25 \%$ en respectaient deux et moins de $3 \%$ respectaient les trois recommandations et se conformaient de ce fait aux Directives en matière de mouvement sur 24 heures.

Le tableau 4 présente les proportions de participants respectant diverses combinaisons de ces recommandations. Moins de $5 \%$ respectaient la recommandation pour le temps passé devant un écran seulement et la combinaison des recommandations pour le sommeil et le temps passé devant un écran ainsi que celle pour l'APIME et le temps passé devant un écran. Les pourcentages de participants respectant la recommandation pour le sommeil seulement, pour l'APIME seulement ainsi que la combinaison de recommandations pour le sommeil et l'APIME étaient tous supérieurs à $10 \%$.

\section{Analyse}

Notre étude a permis de déterminer le pourcentage de jeunes Canadiens de 10 à 17 ans respectant les recommandations des Directives en matière de mouvement sur 24 heures : un faible pourcentage (3\%) des jeunes de notre échantillon respectaient les trois recommandations clés. À la lumière des données autodéclarées, il semble que davantage d'enfants et de jeunes respectent la recommandation pour le sommeil (66\%) que celle pour l'APIME (35\%) et celle pour le temps passé devant un écran (8\%).

Les Directives en matière de mouvement sur 24 heures publiées récemment sont les premières lignes directrices de santé publique à intégrer plusieurs comportements relatifs au mouvement. Aucune 
TABLEAU 2

Pourcentage de jeunes de 10 à 17 ans qui respectent les Directives en matière de mouvement sur 24 heures, par recommandation respectée

\begin{tabular}{|c|c|c|c|c|c|}
\hline \multirow{3}{*}{ Recommandation } & \multirow{3}{*}{$\begin{array}{c}\text { Tous les } \\
\text { participants } \\
\text { (n = } 22115 \text { ) } \\
\text { \% (IC à } 95 \%)\end{array}$} & \multicolumn{2}{|c|}{ Sexe } & \multicolumn{2}{|c|}{ Groupe d'âge } \\
\hline & & $\begin{array}{c}\text { Garçons } \\
(n=10465)\end{array}$ & $\begin{array}{c}\text { Filles } \\
(n=11650)\end{array}$ & $\begin{array}{l}10 \text { à } 13 \text { ans } \\
(n=10236)\end{array}$ & $\begin{array}{l}14 \text { à } 17 \text { ans } \\
(n=11879)\end{array}$ \\
\hline & & \% (IC à $95 \%)$ & \% (IC à $95 \%)$ & \% (IC à $95 \%)$ & \% (IC à $95 \%)$ \\
\hline $\begin{array}{l}\text { Durée du } \\
\text { sommeil }\end{array}$ & $\begin{array}{c}66,2 \\
(64,7 \text { à } 67,7)\end{array}$ & $\begin{array}{c}65,8 \\
(64,1 \text { à 67,5) }\end{array}$ & $\begin{array}{c}66,6 \\
(64,7 \text { à 68,5) }\end{array}$ & $\begin{array}{c}64,5 \\
(62,4 \text { à 66,6) }\end{array}$ & $\begin{array}{c}67,7 \\
(65,9 \text { à 69,5) }\end{array}$ \\
\hline $\begin{array}{l}\text { Activité } \\
\text { physique }\end{array}$ & $\begin{array}{c}35,4 \\
(32,8 \text { à } 38,0)\end{array}$ & $\begin{array}{c}41,6 \\
(38,7 \text { à } 44,6)\end{array}$ & $\begin{array}{c}29,8 \\
(27,1 \text { à } 32,6)^{*}\end{array}$ & $\begin{array}{c}36,0 \\
(33,7 \text { à 38,2) }\end{array}$ & $\begin{array}{c}34,9 \\
(31,2 \text { à } 38,9)\end{array}$ \\
\hline $\begin{array}{l}\text { Temps passé } \\
\text { devant un écran }\end{array}$ & $\begin{array}{c}8,1 \\
(7,1 \text { à } 9,0)\end{array}$ & $\begin{array}{c}7,1 \\
(6,1 \text { à } 8,1)\end{array}$ & $\begin{array}{c}8,9 \\
(7,7 \text { à } 10,1)^{*}\end{array}$ & $\begin{array}{c}11,6 \\
(10,3 \text { à } 12,9)\end{array}$ & $\begin{array}{c}5,0 \\
(4,3 \text { à } 5,8)^{* *}\end{array}$ \\
\hline
\end{tabular}

Abréviations : Directives en matière de mouvement sur 24 heures, Directives canadiennes en matière de mouvement sur 24 heures pour les enfants et les jeunes : une approche intégrée regroupant l'activité physique, le comportement sédentaire et le sommeil; IC, intervalle de confiance.

Remarque : Les données présentées sont des prévalences (IC à $95 \%$ ).

"Significativement différent chez les garçons $(p<0,05)$.

"Significativement différent chez les jeunes de 10 à 13 ans $(p<0,05)$. étude de surveillance antérieure n’a donc analysé le respect simultané des recommandations pour le sommeil, pour l'activité physique et pour le temps passé devant un écran, ce qui fait que les résultats de notre étude sont nouveaux et ne peuvent de ce fait être directement comparés à ceux d'études précédentes. Ceci dit, ces études reposaient sur des échantillons représentatifs à l'échelle nationale de jeunes Canadiens et portaient sur le respect individuel des recommandations des Directives, ce qui nous permet d'en discuter ci-dessous.
Les lignes directrices canadiennes précédentes sur l'activité physique pour les jeunes d'âge scolaire recommandaient 60 minutes d'APIME chaque jour? ${ }^{7}$. Cette recommandation est la même que celle des Directives en matière de mouvement sur 24 heures. Dans une optique de surveillance, les chercheurs ont habituellement considéré les jeunes faisant 60 minutes d'APIME 6 ou 7 jours par semaine comme physiquement actifs ${ }^{23}$. Le changement de paradigme des nouvelles Directives en matière de mouvement sur 24 heures (et les recommandations de
TABLEAU 3

Pourcentage de jeunes de 10 à 17 ans qui respectent les Directives en matière de mouvement sur 24 heures, en fonction du nombre de recommandations respectées

\begin{tabular}{|c|c|c|c|c|c|}
\hline \multirow{3}{*}{$\begin{array}{l}\text { Nombre de } \\
\text { recommandations } \\
\text { respectées }\end{array}$} & \multirow{3}{*}{$\begin{array}{c}\text { Tous les } \\
\text { participants } \\
(n=22115) \\
\% \text { (IC à } 95 \%)\end{array}$} & \multicolumn{2}{|c|}{ Sexe } & \multicolumn{2}{|c|}{ Groupe d'âge } \\
\hline & & $\begin{array}{c}\text { Garçons } \\
(n=10465)\end{array}$ & $\begin{array}{c}\text { Filles } \\
(n=11650)\end{array}$ & $\begin{array}{c}10 \text { à } 13 \text { ans } \\
(n=10236)\end{array}$ & $\begin{array}{c}14 \text { à } 17 \text { ans } \\
(n=11 \text { 879) }\end{array}$ \\
\hline & & $\%$ (IC à $95 \%)$ & \% (IC à $95 \%)$ & \% (IC à $95 \%)$ & \% (IC à $95 \%)$ \\
\hline Aucune & $\begin{array}{c}20,9 \\
(19,5 \text { à } 22,3)\end{array}$ & $\begin{array}{c}18,8 \\
(17,3 \text { à } 20,4)\end{array}$ & $\begin{array}{c}22,9 \\
(21,1 \text { à } 24,4)^{*}\end{array}$ & $\begin{array}{c}21,2 \\
(19,4 \text { à } 23,0)\end{array}$ & $\begin{array}{c}20,7 \\
(19,0 \text { à } 22,3)\end{array}$ \\
\hline Une & $\begin{array}{c}51,1 \\
(49,4 \text { à 52,9) }\end{array}$ & $\begin{array}{c}50,3 \\
(48,5 \text { à } 52,0)\end{array}$ & $\begin{array}{c}51,9 \\
(49,6 \text { à 54,2) }\end{array}$ & $\begin{array}{c}49,3 \\
(47,6 \text { à } 50,9)\end{array}$ & $\begin{array}{c}52,7 \\
(50,4 \text { à } 55,1)^{* *}\end{array}$ \\
\hline Deux & $\begin{array}{c}25,3 \\
(23,5 \text { à } 27,2)\end{array}$ & $\begin{array}{c}28,4 \\
(26,1 \text { à } 30,8)\end{array}$ & $\begin{array}{c}22,6 \\
(20,7 \text { à } 24,4)^{*}\end{array}$ & $\begin{array}{c}25,9 \\
(24,2 \text { à } 27,6)\end{array}$ & $\begin{array}{c}24,8 \\
(22,1 \text { à } 27,6)\end{array}$ \\
\hline Les trois & $\begin{array}{c}2,6 \\
(2,2 \text { à } 3,0)\end{array}$ & $\begin{array}{c}2,5 \\
(2,0 \text { à } 3,0)\end{array}$ & $\begin{array}{c}2,7 \\
(2,2 \text { à } 3,3)\end{array}$ & $\begin{array}{c}3,7 \\
(3,0 \text { à } 4,3)\end{array}$ & $\begin{array}{c}1,7 \\
(1,3 \text { à } 2,2)^{* *}\end{array}$ \\
\hline
\end{tabular}

Abréviations : Directives en matière de mouvement sur 24 heures, Directives canadiennes en matière de mouvement sur 24 heures pour les enfants et les jeunes : une approche intégrée regroupant l'activité physique, le comportement sédentaire et le sommeil; IC, intervalle de confiance.

Remarque : Les données présentées sont des prévalences (IC à $95 \%$ ).

"Significativement différent chez les garçons $(p<0,05)$.

"Significativement différent chez les jeunes âgés de 10 à 13 ans $(p<0,05)$. surveillance qu'elles contiennent) est qu'il faut calculer la moyenne d'APIME quotidienne sur une semaine complète ${ }^{14}$. Les données autodéclarées des cycles 2001-2002, 2005-2006, 2009-2010 et 2013-2014 de l'ECSEAS canadienne laissent penser qu'entre $18 \%$ et $20 \%$ des enfants de $6^{\text {e }}$ année au secondaire 4 respectent la cible de 60 minutes chaque jour de la semaine ${ }^{16}$. Le changement de paradigme permet de tenir compte du fait qu'il existe chez les jeunes une variabilité entre les journées quant à l'APIME et aux autres comportements en matière de mouvement et qu'au fond, il est plus important d'accumuler un volume suffisant d'APIME sur une semaine que d'accumuler une durée précise chaque jour $^{1,2}$.

Les lignes directrices canadiennes précédentes sur le comportement sédentaire chez les jeunes d'âge scolaire recommandaient un maximum de 2 heures par jour de temps de loisir passé devant un écran ${ }^{26}$. Cette recommandation est la même que celle des Directives en matière de mouvement sur 24 heures. Les études canadiennes précédentes de surveillance sur le temps passé devant un écran prenaient traditionnellement en compte la moyenne quotidienne de temps d'écran et n'exigeaient pas que la cible des 2 heures soit respectée chaque jour de la semaine ou la majorité des jours de la semaine. Cette stratégie est cohérente avec les recommandations de surveillance pour le temps passé devant un écran des nouvelles Directives en matière de mouvement sur 24 heures $^{14}$. Les résultats du cycle 2009-2010 de l'ECSEAS indiquent que $19 \%$ des jeunes de $6^{e}$ année au secondaire 4 respectaient la recommandation pour le temps passé devant un écran ${ }^{16}$, ce qui est deux fois plus élevé que le niveau de prévalence déclaré ( $8 \%$ ) pour le cycle 2013-2014. Des changements considérables ont été apportés aux questions portant sur le temps passé devant un écran entre 2009-2010 et 2013-2014 dans l'ECSEAS, ce qui peut avoir contribué à cette grande différence. Plus précisément, les questions ont été modifiées afin de tenir compte de l'évolution de la technologie, comme l'utilisation des tablettes pour regarder des émissions, la consultation des médias sociaux à l'ordinateur, l'inclusion des disques Blu-Ray en plus des DVD pour les films, l'inclusion des vidéos YouTube ou d'une autre plateforme similaire et l'exclusion des jeux vidéo actifs.

Nous disposons d'une quantité limitée de données de surveillance sur la durée du 
TABLEAU 4

Pourcentage de jeunes de 10 à 17 ans qui respectent les Directives en matière de mouvement sur 24 heures, en fonction des combinaisons de recommandations respectées

\begin{tabular}{|c|c|c|c|c|c|}
\hline \multirow{3}{*}{$\begin{array}{l}\text { Combinaison de } \\
\text { recommandations } \\
\text { respectées }\end{array}$} & \multirow{3}{*}{ 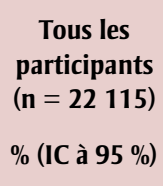 } & \multicolumn{2}{|c|}{ Sexe } & \multicolumn{2}{|c|}{ Groupe d'âge } \\
\hline & & $\begin{array}{c}\text { Garçons } \\
(n=10465)\end{array}$ & $\begin{array}{c}\text { Filles } \\
(\mathrm{n}=11650)\end{array}$ & $\begin{array}{c}10 \text { à } 13 \text { ans } \\
(n=10236)\end{array}$ & $\begin{array}{l}14 \text { à } 17 \text { ans } \\
(n=11 \text { 879) }\end{array}$ \\
\hline & & \% (IC à $95 \%)$ & \% (IC à $95 \%)$ & \% (IC à $95 \%)$ & \% (IC à $95 \%)$ \\
\hline Aucune & $\begin{array}{c}20,9 \\
(19,5 \text { à 22,3) }\end{array}$ & $\begin{array}{c}18,8 \\
(17,3 \text { à } 20,4)\end{array}$ & $\begin{array}{c}22,7 \\
(21,1 \text { à } 24,4)^{*}\end{array}$ & $\begin{array}{c}21,1 \\
(19,4 \text { à } 23,0)\end{array}$ & $\begin{array}{c}20,7 \\
(19,0 \text { à } 22,3)\end{array}$ \\
\hline $\begin{array}{l}\text { Durée du sommeil } \\
\text { seulement }\end{array}$ & $\begin{array}{c}39,2 \\
(36,9 \text { à } 41,4)\end{array}$ & $\begin{array}{c}35,9 \\
(33,7 \text { à } 38,1)\end{array}$ & $\begin{array}{c}42,1 \\
(39,2 \text { à } 45,0)^{*}\end{array}$ & $\begin{array}{c}36,1 \\
(34,3 \text { à } 37,9)\end{array}$ & $\begin{array}{c}41,8 \\
(38,6 \text { à } 45,0)^{* *}\end{array}$ \\
\hline $\begin{array}{l}\text { Activité physique } \\
\text { seulement }\end{array}$ & $\begin{array}{c}10,7 \\
(9,7 \text { à } 11,6)\end{array}$ & $\begin{array}{c}13,2 \\
(12,0 \text { à } 14,4)\end{array}$ & $\begin{array}{c}8,4 \\
(7,3 \text { à } 9,4)^{*}\end{array}$ & $\begin{array}{c}11,4 \\
(10,4 \text { à } 12,5)\end{array}$ & $\begin{array}{c}10,0 \\
(8,7 \text { à } 11,3)\end{array}$ \\
\hline $\begin{array}{l}\text { Temps d'écran } \\
\text { seulement }\end{array}$ & $\begin{array}{c}1,3 \\
(1,0 \text { à } 1,5)\end{array}$ & $\begin{array}{c}1,1 \\
(0,8 \text { à } 1,4)\end{array}$ & $\begin{array}{c}1,4 \\
(1,1 \text { à } 1,8)\end{array}$ & $\begin{array}{c}1,7 \\
(1,3 \text { à } 2,1)\end{array}$ & $\begin{array}{c}1,0 \\
(0,7 \text { à } 1,2)^{* *}\end{array}$ \\
\hline $\begin{array}{l}\text { Durée du sommeil } \\
\text { + activité physique }\end{array}$ & $\begin{array}{c}21,8 \\
(19,3 \text { à } 23,0)\end{array}$ & $\begin{array}{c}24,9 \\
(22,4 \text { à } 27,3)\end{array}$ & $\begin{array}{c}17,9 \\
(16,1 \text { à } 19,6)^{*}\end{array}$ & $\begin{array}{c}19,6 \\
(18,9 \text { à } 21,3)\end{array}$ & $\begin{array}{c}22,5 \\
(19,8 \text { à } 25,2)^{* *}\end{array}$ \\
\hline $\begin{array}{l}\text { Durée du sommeil } \\
\text { + temps d'écran }\end{array}$ & $\begin{array}{c}3,2 \\
(2,3 \text { à } 3,7)\end{array}$ & $\begin{array}{c}2,5 \\
(2,0 \text { à } 3,0)\end{array}$ & $\begin{array}{c}3,9 \\
(3,2 \text { à } 4,5)^{*}\end{array}$ & $\begin{array}{c}5,0 \\
(4,3 \text { à } 5,9)\end{array}$ & $\begin{array}{c}1,7 \\
(1,4 \text { à } 2,0)^{* *}\end{array}$ \\
\hline $\begin{array}{l}\text { Activité physique } \\
\text { + temps d'écran }\end{array}$ & $\begin{array}{c}0,9 \\
(0,7 \text { à } 1,1)\end{array}$ & $\begin{array}{c}1,0 \\
(0,8 \text { à } 1,3)\end{array}$ & $\begin{array}{c}0,8 \\
(0,6 \text { à } 1,0)\end{array}$ & $\begin{array}{c}1,2 \\
(0,9 \text { à } 1,5)\end{array}$ & $\begin{array}{c}0,7 \\
(0,4 \text { à } 0,9)^{* *}\end{array}$ \\
\hline Les trois & $\begin{array}{c}2,6 \\
(2,2 \text { à } 3,0)\end{array}$ & $\begin{array}{c}2,5 \\
(2,0 \text { à } 3,0)\end{array}$ & $\begin{array}{c}2,7 \\
(2,2 \text { à } 3,3)\end{array}$ & $\begin{array}{c}3,7 \\
(3,0 \text { à } 4,3)\end{array}$ & $\begin{array}{c}1,7 \\
(1,3 \text { à } 2,2)^{* *}\end{array}$ \\
\hline
\end{tabular}

Abréviations : Directives en matière de mouvement sur 24 heures, Directives canadiennes en matière de mouvement sur 24 heures pour les enfants et les jeunes : une approche intégrée regroupant l'activité physique, le comportement sédentaire et le sommeil; IC, intervalle de confiance.

Remarque : Les données présentées sont des prévalences (IC à $95 \%$ ).

Significativement différent chez les garçons $(p<0,05)$.

"Significativement différent chez les jeunes de 10 à 13 ans $(p<0,05)$.

sommeil. À notre connaissance, les seules données canadiennes comparables proviennent de l'Enquête canadienne sur les mesures de la santé de 2012-2013. Les résultats de cette enquête laissent penser que $18 \%$ des enfants de 5 à 11 ans et $33 \%$ des jeunes de 12 à 17 ans ne dorment pas suffisamment ${ }^{27}$. La durée appropriée de sommeil a été définie comme suit : de 10 à 13 heures par nuit pour les enfants de 5 ans, de 9 à 11 heures par nuit pour les enfants de 6 à 13 ans et de 8 à 10 heures par nuit pour les jeunes de 14 à 17 ans. Ces résultats sont similaires à ceux de notre étude, qui laisse penser que $35 \%$ des jeunes de 10 à 13 ans et $32 \%$ des jeunes de 14 à 17 ans ne dorment pas suffisamment.

L'une des conclusions que notre étude permet de tirer est qu'une très faible proportion (moins de $3 \%$ ) des Canadiens de 10 à 16 ans respectent les trois recommandations des nouvelles Directives canadiennes en matière de mouvement sur 24 heures pour les enfants et les jeunes. L'un des constats à retenir est que mettre l'accent sur un seul comportement en matière de mouvement ne permet pas de saisir l'envergure de la crise relative liée au mouvement qu'on observe chez les jeunes
Canadiens : par exemple, seulement un tiers de l'échantillon ne respectait pas la durée recommandée de sommeil. Par ailleurs, il demeure utile d'analyser les comportements en matière de mouvement de façon individuelle et pas simplement en combinaisons, pour cerner les comportements les plus problématiques. Dans notre étude, les résultats pour le temps passé devant un écran sont ainsi particulièrement préoccupants : seulement $8 \%$ des participants respectaient la recommandation correspondante. Les interventions aptes à réduire efficacement le temps passé devant un écran peuvent de ce fait se révéler particulièrement utiles pour améliorer le profil de mouvement des jeunes Canadiens.

\section{Forces et limites}

Une limitation importante de notre étude est l'autodéclaration des données sur le comportement. Elle peut avoir entraîné une mauvaise classification comme une sous-estimation ou une surestimation des durées quant au respect des recommandations. Il faut aussi souligner que l'évaluation de l'APIME était réduite aux activités effectuées en classe et pendant les temps libres à l'extérieur de l'école ainsi qu'au transport actif jusqu'à l'école ou pour en revenir. Le transport actif vers d'autres destinations et les activités effectuées à l'école pendant les récréations et à l'heure du dîner n’ont pas été pris en considération. De plus, l'évaluation du temps passé devant un écran comprenait les devoirs (dans la question sur l'utilisation d'un ordinateur), alors que la recommandation de 2 heures ou moins par jour de temps d'écran porte spécifiquement sur le temps de loisir passé devant un écran ${ }^{14}$. La méthode de collecte a fait en sorte qu'il manquait beaucoup de données, car plusieurs participants ont choisi de ne pas répondre à plusieurs questions ou n'ont pas eu le temps de le faire. Ces participants ont été exclus des analyses, ce qui peut avoir biaisé les résultats dans la mesure où les comportements en matière de mouvement seraient différents entre participants inclus et exclus. Une autre limitation de l'étude relève de la stratégie d'échantillonnage, qui excluait les jeunes inscrits à un programme pour enfants ayant des besoins particuliers, ceux vivant dans une réserve ou ne fréquentant pas une école publique ( $p$. ex. enfant sans-abri). Bien que ces jeunes ne représentent que moins de $7 \%$ des Canadiens de la tranche d'âge ciblée, ils figurent parmi les enfants canadiens les plus vulnérables et leurs comportements en matière de mouvement sont susceptibles d'être différents de ceux présentés dans le cadre de notre étude. Des travaux de recherche supplémentaires sur ces groupes vulnérables sont nécessaires.

\section{Conclusion}

Si $79 \%$ des Canadiens de 10 à 17 ans respectent une ou plusieurs des recommandations des Directives canadiennes en matière de mouvement sur 24 heures pour les enfants et les jeunes, seulement $3 \%$ respectent l'ensemble des trois recommandations. Ce respect est particulièrement faible pour les recommandations relatives au temps passé devant un écran et l'APIME. Nous espérons que l'information recueillie dans le cadre de notre étude sera utile pour établir des programmes et des politiques visant à promouvoir des comportements sains en matière de mouvement chez les jeunes d'âge scolaire.

\section{Remerciements}

L'ECSEAS canadienne de 2014 et cette étude ont été financées par l'Agence de la 
santé publique du Canada. IJ a aussi reçu un prix du Programme des chaires de recherche du Canada.

\section{Conflits d'intérêts}

Les auteurs déclarent n'avoir aucun conflit d'intérêts.

\section{Contributions des auteurs et avis}

Tous les auteurs ont contribué à la conception de l'étude et en ont approuvé la version définitive. IJ a effectué les analyses statistiques et a rédigé la première ébauche du manuscrit. KR et WT ont participé à l'interprétation des résultats et effectué une révision critique du manuscrit.

Le contenu de cet article et les opinions qui y sont exprimées n'engagent que les auteurs et ne représentent pas forcément les opinions du Gouvernement du Canada.

\section{Références}

1. Janssen I, Leblanc AG. Systematic review of the health benefits of physical activity and fitness in school-aged children and youth. Int J Behav Nutr Phys Act. 2010;7(1):40.

2. Poitras V, Gray C, Borghese M, et al. Systematic review of the relationships between physical activity and health indicators in school-aged children and youth. Appl Physiol Nutr Metab. 2016;41(6):S197-S239.

3. LeBlanc AG, Spence J, Carson V, et al. Systematic review of sedentary behaviour and health indicators in the early years (aged 0-4 years). Appl Physiol Nutr Metab. 2012;37:753-772.

4. Carson V, Hunter S, Kuzik N, et al. Systematic review of the relationships between sedentary behaviour and health indicators in school-aged children and youth: an update. Appl Physiol Nutr Metab. 2016;41(6 Suppl 3):S240-S65

5. Gruber R, Carrey N, Weiss SK, et al. Position statement on pediatric sleep for psychiatrists. J Can Acad Child Adolesc Psychiatry. 2014;23(3):174-195.

6. Chaput JP, Gray C, Poitras V, et al. Systematic review of the relationships between sleep duration and health indicators in school-aged children and youth. Appl Physiol Nutr Metab. 2016;41(6 Suppl 3):S266-S282.
7. Tremblay MS, Warburton DE, Janssen I, et al. New Canadian physical activity guidelines. Appl Physiol Nutr Metab. 2011;36(1):36-46.

8. Tremblay MS, Leblanc AG, Janssen I, et al. Canadian sedentary behaviour guidelines for children and youth. Appl Physiol Nutr Metab. 2011;36(1): 59-64;65-71.

9. Hirshkowitz M, Whiton K, Albert SM, et al. National Sleep Foundation's sleep time duration recommendations: methodology and results summary. Sleep Health. 2015;1:40-43.

10. Chastin SF, Palarea-Albaladejo J, Dontje ML, Skelton DA. Combined effects of time spent in physical activity, sedentary behaviors and sleep on obesity and cardio-metabolic health markers: A novel compositional data analysis approach. PloS One. 2015; 10(10):e0139984.

11. Buman MP, Winkler EA, Kurka JM, et al. Reallocating time to sleep, sedentary behaviors, or active behaviors: associations with cardiovascular disease risk biomarkers, NHANES 20052006. Am J Epidemiol. 2014;179(3): 323-334.

12. Chaput JP, Carson V, Gray CE, Tremblay MS. Importance of all movement behaviors in a 24-hour period for overall health. International journal of environmental research and public health. 2014;11(12):12575-12581.

13. Saunders TJ, Gray CE, Poitras V, et al. Combinations of physical activity, sedentary behaviour and sleep: relationships with health indicators in school-aged children and youth. Appl Physiol Nutr Metab. 2016;41(6 Suppl 3):S283-S93.

14. Tremblay MS, Carson V, Chaput JP, et al. Canadian 24-Hour Movement Guidelines for Children and Youth: an integration of physical activity, sedentary behaviour, and sleep. Appl Physiol Nutr Metab. 2016;41(6 Suppl 3):S311-S327.

15. Currie C, Nic Gabhainn S, Godeau E. The Health Behaviour in School-aged Children: WHO Collaborative CrossNational (HBSC) study: origins, concept, history and development 1982-2008. Int J Public Health. 2009;54 Suppl 2:131-139.
16. Freeman JG, King M, Pickett W, éditeurs. La santé des jeunes Canadiens : un accent sur la santé mentale. Ottawa (Ontario) : Agence de la santé publique du Canada; 2012.

17. Wolfson AR, Carskadon MA, Acebo C, et al. Evidence for the validity of a sleep habits survey for adolescents. Sleep. 2003;26(2):213-216.

18. Janssen I. Active play: an important physical activity strategy in the fight against childhood obesity. Can J Public Health. 2014;105(1):e22-e27.

19. Roberts C, Alexander D, Currie D, Haug E, Komkov A, Tynajala J. Physical activity. Health Behaviour in School-aged Children (HBSC): Research protocol for the 2005/06 survey. Edinburgh : Child and Adolescent Health Research Unit; 2006.

20. Booth ML, Okely AD, Chey T, Bauman A. The reliability and validity of the physical activity questions in the WHO health behaviour in school children (HBSC) survey: a population study. Br J Sports Med. 2001;35(4): 263-267.

21. Brener ND, Collins JL, Kann L, Warren CW, Williams BI. Reliability of the Youth Risk Behavior Survey Questionnaire. Am J Epidemiol. 1995;141(6): 575-580

22. ParticipACTION. Garder les enfants à l'intérieur : un plus grand risque! Bulletin de l'activité physique chez les jeunes de ParticipACTION 2015. Toronto (Ontario) : ParticipACTION; 2015.

23. Colley RC, Garriguet D, Janssen I, Craig CL, Clarke J, Tremblay MS. Activité physique des enfants et des jeunes au Canada : résultats d'accélérométrie de l'Enquête canadienne sur les mesures de la santé de 2007-2009. Rapports sur la santé. 2011;22(1):1-10.

24. Organisation mondiale de la santé (OMS). Recommandations mondiales en matière d'activité physique pour la santé. [Internet]. Genève $(\mathrm{CH})$ : OMS; 2010. En ligne à : www.who.int /dietphysicalactivity/factsheet_ recommendations/fr/ 
25. Rey-Lopez JP, Vicente-Rodriguez G, Ortega FB, et al. Sedentary patterns and media availability in European adolescents: The HELENA study. Prev Med. 2010;51(1):50-55.

26. Tremblay MS, Leblanc AG, Janssen I, et al. Canadian sedentary behaviour guidelines for children and youth. Appl Physiol Nutr Metab. 2011;36(1): 59-64.

27. Centre de prévention des maladies chroniques, Agence de la santé publique du Canada. Cadre d'indicateurs des maladies chroniques et des blessures : Statistiques rapides, édition 2015. Promotion de la santé et prévention des maladies chroniques au Canada. 2015;35(10):208-209. 